\title{
On Routing Protocols for High Performance
}

\author{
Noor H. BHANGWAR ${ }^{1}$, Imtiaz A. HALEPOTO ${ }^{1 *}$, Intesab H. SADHAYO ${ }^{2}$, \\ Suhail KHOKHAR ${ }^{3}$, Asif A. LAGHARI ${ }^{4}$ \\ ${ }^{1}$ Department of Computer Systems Engineering, \\ QUEST, Pakistan \\ e-mail: halepoto@quest.edu.pk (*Corresponding authors) \\ ${ }^{2}$ Department of Telecommunication Engineering, \\ QUEST, Pakistan \\ ${ }^{3}$ Department of Electrical Engineering, \\ QUEST, Pakistan \\ ${ }^{4}$ School of Computer Science \& Technology, \\ Harbin Institute of Technology, China
}

\begin{abstract}
In computer networks, the routing protocols play the main role in source to destination delivery of packets with minimum communication time between the nodes as well as in selecting the best paths while utilizing fewer resources. The design of an efficient routing protocol almost in any kind of network is an important issue specifically in the Mobile Ad Hoc Networks (MANETs). Recently, the network research community has been very active towards efficient routing over MANETs in order to provide high-performance network services. MANET is a collection of autonomous and infrastructureless wireless mobile nodes that communicate with each other by dynamically forming a multi-hop radio network. The highly dynamic and frequent changes in topology within MANETs raise many issues in the performance of routing protocols. So choosing an efficient routing protocol in MANETs is a challenging task. This study evaluates the performance of some of the well-known routing protocols that benefit the network function virtualization (NFV) in the cloud computing environment. The protocols used are AODV, DSDV, OLSR and GPSR. The proposed scenarios for the simulation of the routing protocols include a varying number of moving nodes. In the investigations of up to 50 nodes, the OLSR outperforms AODV, DSDV and GPSR.
\end{abstract}

Keywords: Routing protocols, Mobile ad hoc networks, Wireless links, Cloud computing.

\section{Introduction}

The Mobile Ad hoc Network (MANET) [8] is an auto-configurable and self-organized wireless network that possesses any infrastructure for the communication of nodes. Nodes in MANET can communicate directly with each other without any centralized controller or access point over a dynamic topology. One of the most popular variant of MANET is Vehicular ad hoc network (VANET) [14], which reduces the traffic accidents by providing intelligent communication between vehicles and the fixed traffic management system. There are many applications where MANETs are needed, such as defensive battlefields, sensor networks, reaching out flood hit areas and emergency health services. Moreover, with the introduction of NFV, most of the network load and processing is moving towards the cloud computing.

In all these applications, MANETs are composed of the bandwidth constrained wireless links where mobile nodes maintain the connectivity in a decentralized manner. The link and node failures in such scenarios are very common. Due to which the nodes select an alternate path for successful data transmission. Mobile nodes in MANETs can receive and forward packets while playing the role as a router. Mobile nodes in MANET must be within the range of influence of the transmission. Additionally, MANETs are usually composed of devices that are limited in power as well cover short range for communication. So, in most cases mobile nodes follow a transit way of communication. The routing protocols in MANETs provide an efficient way of communication by selecting the best paths from a source to destination.

There are three categories of routing protocols based on the characteristics, which are classified as proactive, reactive and hybrid. Proactive category of MANET includes those protocols that always contain the routing information of all connected nodes that the part of the network. These protocols maintain a routing table. The routing table is a data structure that contains the routing information of all of the nodes. Reactive protocols establish a route only on demand by broadcasting a special request packet on the network. Hybrid protocols possess the routing capabilities of both proactive and reactive. Routes in hybrid protocols are found quickly in the routing zone. The new category of routing is position based where the actual location 
coordinates are used to communicate the source and destination nodes.

The design of a routing protocol with focus on its efficiency in any network, especially in wireless MANETs, is a challenge. Several routing protocols have been proposed. Among them, the most prominent protocols are Ad hoc On-demand Distance Vector (AODV) [18], Destination Sequenced Distance Vector (DSDV) [19], Optimized Link State Routing (OLSR) [16] and Greedy Perimeter Stateless Routing (GPSR) [10]. In this paper, the performance of AODV, DSDV, OLSR and GPSR is compared over a wireless scenario. Through analysis it is concluded that OLSR and GPSR are prominent protocols in terms of performance as compared to DSDV and AODV. However, in case of higher mobility OLSR outperforms the GPSR.

The rest of the paper is organized as follows. In Section 2 related work is presented. Both section 3 and 4 provides an overview of the protocols used for this study. The details of experimentation are presented in Section 5. The discussion on results is presented in Section 6. In Section 7 a brief conclusion is given, and some future work is proposed.

\section{Related Work}

Many authors had studied and evaluated the routing protocols for MANET using different scenarios and parameters [16, 9-14, 24].

In $[1,2], A O D V$, DSDV and GPSR routing protocols in MANET are analyzed by using packet delivery ratio, average end-to-end delay and normalized load parameters. They observed that the performance of GPSR routing protocol decreases when nodes move in high speed. The findings also show that AODV has long delay because it takes relatively large time to find the destination node. Unlike, DSDV has the short delay because routes are always immediately available. In [3], AODV, DSR (dynamic source routing), OLSR, GRP (gateway routing protocol) and TORA (temporary ordered routing algorithm) were analyzed by using throughput, delay, load and packet drop parameters over video conferencing and email using high bandwidth and data rate. According to their suggestion, AODV is slightly preferable for video conferencing applications. OLSR and GRP are suggested as suitable for e-mail applications. In a similar study in [4], for the evaluation of AODV, DSR, TORA and GRP the authors analyzed that OLSR outperforms the AODV, DSR, TORA and GRP. However, the investigations on routing overhead encourage the use of GPSR [13]. The work in $[22,17]$ compared AODV and OLSR over a scenario of 30 nodes. Where, the results indicate the OLSR outperforms AODV.

The study in [23] for mobile and stationary nodes suggests AODV for efficient routing over DSDV and DSR. A study on mobile and stationary nodes with perspective of energy efficiency and collision is done in [11]. Their findings show that LAR1 (location aided routing 1) protocol suffers from collision and WRP (wireless routing protocol) consumes maximum energy. In terms of routing overhead, the study in [24] finds DSR as an efficient protocol over scenario of varying packet size and mobility. The work in [20] recommended the AODV protocol when compared to DSDV for UDP traffic. The work in [6] investigated the effect of varying node speed on the routing protocol. The analysis shows that MDSDV (multipath DSDV) achieves similar throughput as of AODV. The investigations from the study in [12] highlight that OLSR works well with the dense networks.

\section{Routing Protocols for MANETs}

Routing protocols in MANET are classified into three categories:

\subsection{Flat Ad hoc Routing}

Flat Ad hoc routing includes those protocols that do not setup hierarchies with the cluster of nodes. All nodes in the topology perform the similar tasks for the routing. The addressing scheme is flat. Flat routing protocols are further classified as pro-active, reactive and hybrid.

1) Proactive Routing Protocols: These Protocols always maintain up-to-date routing information of all nodes in the network. The protocols keep the information of all connected nodes in the form of tables all the time. These tables are further shared with their neighbors. In case of any change in the network topology, every node updates the routing table. Proactive routing protocols consume network bandwidth. The most widely used proactive protocols are DSDV, OLSR and Wireless Routing Protocol (WRP). 
2) Reactive Routing Protocols: These protocols establish a route only on demand. The protocol begins the route discovery only when the node needs to communicate with another node in the network. Reactive routing protocols reduce the network overhead. AODV, DSR, and TORA are the famous protocols of this category.

3) Hybrid Routing Protocols: These protocols combine the features of proactive and reactive routing. The protocols locally behave as proactive and globally as reactive. The convergence time of hybrid protocols is short. The examples of hybrid protocols are Zone routing protocol (ZRP) and zone based hierarchical link state protocol (ZHLS).

\subsection{Hierarchical Ad hoc Routing}

For large-scale wireless networks, clustering of nodes can be an efficient solution. The main advantage of this approach is the locality property. If a cluster is established, nodes remain within the cluster. If the topology of the cluster changes, advertisement to the nodes within that cluster is carried out. The nodes of neighboring clusters only need to know the information to reach to the cluster head. A cluster head is a router, which provides communication between to clusters.

\subsection{Geographic Based Routing}

In Geographic based routing protocols, each node knows its own and its neighbor node's geographic position through Geographic Position System (GPS). Information from GPS is used for the routing decision. The well-known position based protocols are GPSR, Geo-cast and Distance Routing Effect Algorithm for Mobility (DREAM).

\section{A Review of AODV, DSDV, OLSR and GPSR}

\subsection{AODV}

AODV is an on-demand routing protocol. Protocols are said to be an on-demand in the sense that the routes are created only when demanded. AODV supports both unicast and multicast routing. For unicast routing, AODV uses different control messages i.e., RREQ (Route Request), RREP (Route Replay), RERR (Route Error) and HELLO. If a node wants to send a packet to another node in the network for which no route is available initially, then the source node starts a route discovery phase and broadcasts a RREQ.
A RREQ includes unique identifier, destination address and sequence number, source address and sequence number and a hop count. A node that receives RREQ, if it is not the destination address then it setup a reverse route to the sender and rebroadcast the updated RREQ. If a subsequent node is the destination, then it creates a RREP. A RREP is a unicast to the origin node along the reverse path. A RREP contains the destination address and sequence number, the source address and sequence number, time-to-live and hope count. When a node receives a RREP, it checks the destination address and sequence number in its own routing table and forwards it accordingly. Once RREP reaches the same node that initiated the RREQ the route request is complete.

\subsection{DSDV}

DSDV is a proactive (table driven) routing protocol. DSDV is based on Bellman Ford Routing Algorithm (BFRA). Information in DSDV protocol is stored in the form of tables. Routing tables are updated periodically and shared with the neighbors. DSDV protocol is an enhancement of distance vector routing. DSDV adds two things to the distance vector algorithm to work in the rapidly changing topology of wireless ad hoc networks, i.e. sequence number and dumping. Each entry in the routing table comes with a sequence number, which may propagate along many paths. Sequence numbers help to avoid loops in networks. Periodic and triggered updates increase the communication overhead in DSDV. Routing in DSDV has the quick convergence and a mechanism to avoid loops, however, it does not support multipath routing.

\subsection{OLSR}

OLSR is also a table-driven protocol. OLSR uses optimized link state routing algorithm. Due to a proactive in nature, the routes in OLSR are immediately available when needed. The key factor of OLSR is the selection of some intermediate nodes called Multipoint Relays (MPRs). The MPR nodes are responsible for forwarding packets in the network. Through MPRs, OLSR reduces the packets retransmission overhead in the network. The performance of OLSR is based on the hop-by-hop routing, which means each node in the network uses its most recent routing information for packets to route. A MPRs covers all the nodes in the network that are 
Table 1. Parameters and Values

\begin{tabular}{|l|l|l|l|l|}
\hline Parameter & Scenario-1 & Scenario-2 & Scenario-3 & Scenario-4 \\
\hline Area & $100 \mathrm{~m} \times 800 \mathrm{~m}$ & $100 \mathrm{~m} \times 800 \mathrm{~m}$ & $100 \mathrm{~m} \times 800 \mathrm{~m}$ & $100 \mathrm{~m} \times 800 \mathrm{~m}$ \\
\hline Simulation Time & $150 \mathrm{sec}$ & $150 \mathrm{sec}$ & $150 \mathrm{sec}$ & $150 \mathrm{sec}$ \\
\hline No. of Nodes & 5 & 10 & 30 & 50 \\
\hline Speed of Nodes & $2 \mathrm{~m} / \mathrm{s}$ & $2 \mathrm{~m} / \mathrm{s}$ & $2 \mathrm{~m} / \mathrm{s}$ & $2 \mathrm{~m} / \mathrm{s}$ \\
\hline Traffic Model & FTP & FTP & FTP & FTP \\
\hline Transport Layer Protocol & TCP & TCP & TCP & TCP \\
\hline Channel & Wireless & Wireless & Wireless & Wireless \\
\hline Bandwidth & $2 \mathrm{Mbps}$ & $2 \mathrm{Mbps}$ & $2 \mathrm{Mbps}$ & $2 \mathrm{Mbps}$ \\
\hline Packet Size & 512 Bytes & 512 Bytes & 512 Bytes & 512 Bytes \\
\hline Mobility Model & Random Way point & Random Way point & Random Way point & Random Way point \\
\hline Propagation Model & Two Ray ground & Two Ray ground & Two Ray ground & Two Ray ground \\
\hline Transmission Range & $250 \mathrm{~m}$ & $250 \mathrm{~m}$ & $250 \mathrm{~m}$ & $250 \mathrm{~m}$ \\
\hline MAC Protocol & 802.11 & 802.11 & 802.11 & 802.11 \\
\hline
\end{tabular}

at-least two hops away. OLSR uses three types of functions, packet forwarding, neighbor sensing, and topology determination. OLSR uses four types of messages, Hello messages, Topology Control (TC) messages, Multiple Interface Declaration (MID) message and Host and Network Association (HNA) messages.

- In order to collect information about the neighboring nodes and links stability in the network, OLSR uses Hello messages.

- In order to broadcast the information about neighbors that includes MPRs selectors list, TC messages are used.

- MID messages are used for advertisement of multiple interfaces.

- HNA messages are used to inform about external routing like network and other addresses.

\subsection{GPSR}

GPSR is used in wireless datagram networks. In order to make the packets forwarding decision, GPSR uses the position of each router. The greedy forwarding decision is done through the use of information of immediate neighbors of the routers. When the packets reaches in a region where greedy forwarding is not possible, then packets are recovered through the use of perimeter routing algorithm. When multiple paths are available to the final destination, GPSR behaves much better in the situation by using the per-router state scheme than the ad hoc routing protocol and shortest path schemes. Whenever any mobility change occurs in the network topology, then the local topology information can be used to find the correct new path rapidly. In GPSR, the location information assigned by the sender is used to identify the destination of each packet in the network. A node can forward a packet by choosing the best greedy next hop, if the position for its neighbor is known. The geographically nearest neighbor to its destination is considered as the best choice for next hop, thus the forwarding process continues sequentially by choosing the nearest geographic hops towards destination. GPSR use beacon mechanism and maintains a location table. The performance of GPSR can be affected due to the beacon loss during weak signals.

\section{Experimental Setup}

Experimental setup of this research is carried out using the Network Simulator 2 (NS2). The advantage of NS2 is that, the implementation of all the required protocols is already available. The simulations are carried out on four different scenarios each with different number of nodes. In each scenario a source node and a destination node is proposed. For example, in Scenario-4, 50 nodes are used, where node 0 is source and node 49 is destination. For each experiment, the throughput, packet delivery ratio and end-toend delay are measured for the comparison and analysis. The simulation of each of the scenario is performed for 10 times in order to record the 


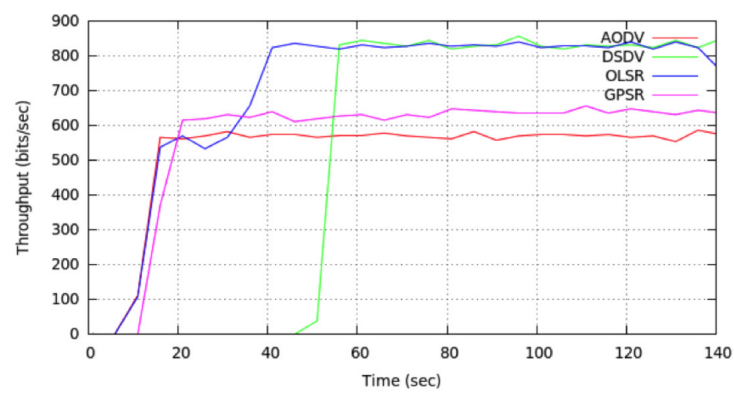

(a) 5 nodes.

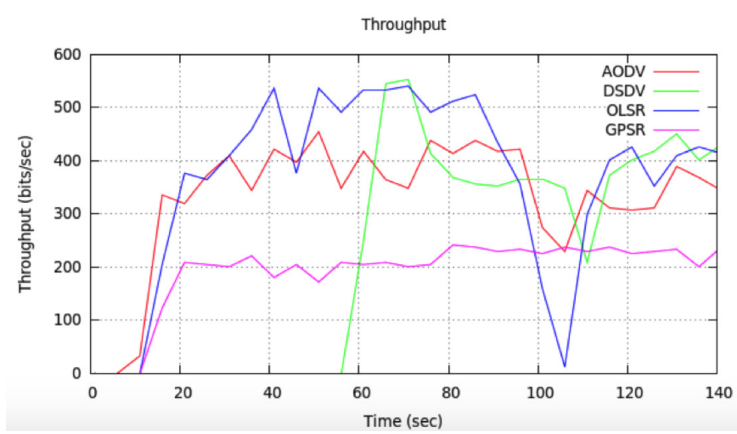

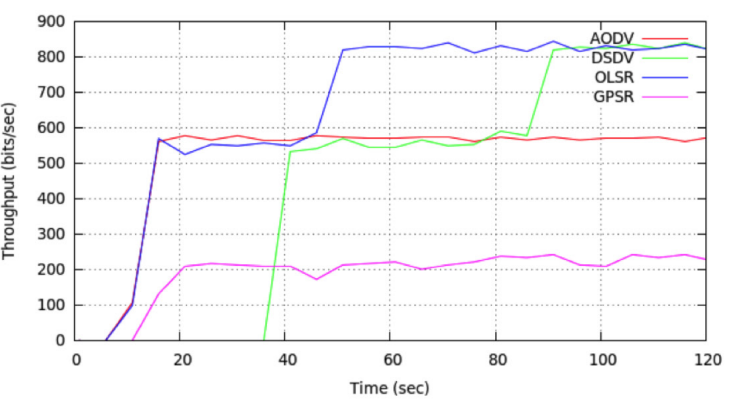

(b) 10 nodes.

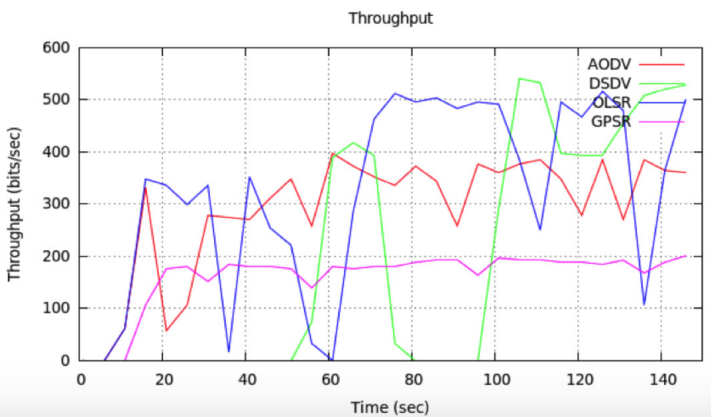

Figure 1. Throughput

reliable results. The final results are averaged for each of the performance measuring parameters. The summary of parameters and their values used for the simulation are shown in Table 1. The rest of the configuration is set on default.

\section{Results and Discussion}

\subsection{Throughput}

Figure 1 shows the throughput of AODV, DSDV, OLSR and GPSR protocols for different number of nodes. The plots show that the throughput of OLSR and DSDV is greater than AODV and GPSR protocols. As we know that OLSR and DSDV are both proactive in nature. In proactive protocols, every node knows its destination already, so their performance in terms of throughput is better than AODV and GPSR protocols, which are reactive and position based in nature. It is also observed that DSDV requires more time for convergence than other protocols (Figure 1(a), (b), (c) and (d)).

The plots also show that the throughput of OLSR is slightly greater than DSDV. It is due the communication overhead of DSDV. For example in Figures 1, approximately first 45 seconds are consumed for the routing table convergence of DSDV. The communication overhead at the beginning of simulation occurs due to the periodic and triggered updates of the routing tables. During the convergence process when the links break in DSDV, each node must wait for an update packet, which consumes time. This wait on each node causes the communication overhead in DSDV. While in OLSR, no such kind of communication overhead is observed except a very short convergence. For that reason, the total throughput of OLSR is greater than DSDV. The plots also show that AODV achieves minimum throughput when compared to other protocols in the given scenario.

\subsection{Packet Delivery Ratio}

The packet delivery ratio of AODV, DSDV, OLSR and GPSR protocols from nodes 5-50 is shown in Figure 2. Graph shows that all routing protocols have similar level of performance in terms of packet delivery ratio. The packet delivery ratio of all protocols AODV, DSDV, OLSR and GPSR is more than $90 \%$ in case of five nodes as shown in Figure 2(a). It is observed that, the packet delivery ratio decrease with the increase in the number of nodes (Figure 2(b), (c) and (d)).

The packet delivery ratio of GPSR increases with the increase in the number of nodes. When the number of node is large and they are mobile, there are sudden changes in the topology. Such changes 


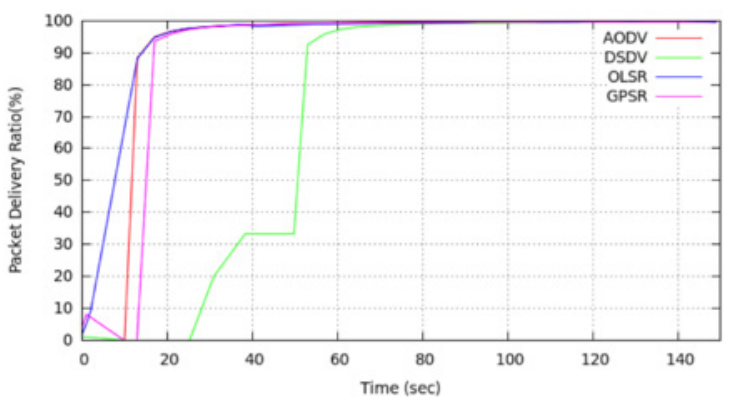

(a) 5 nodes.

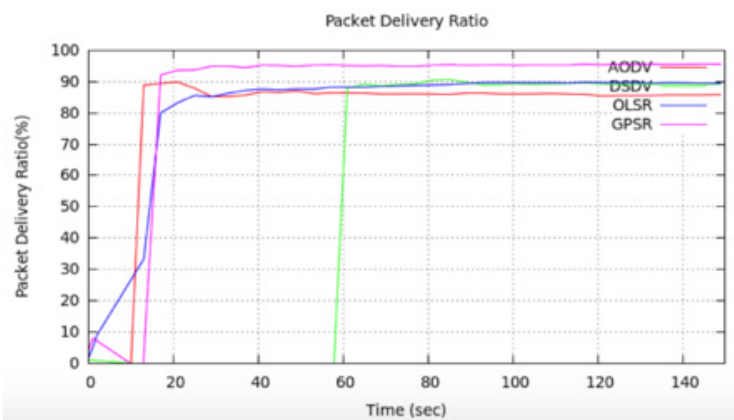

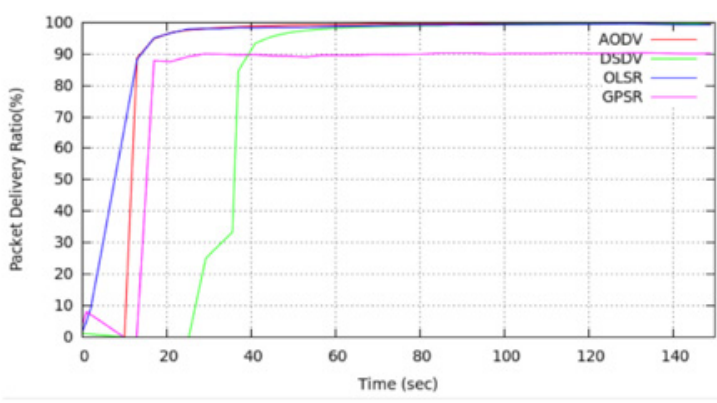

(b) 10 nodes.

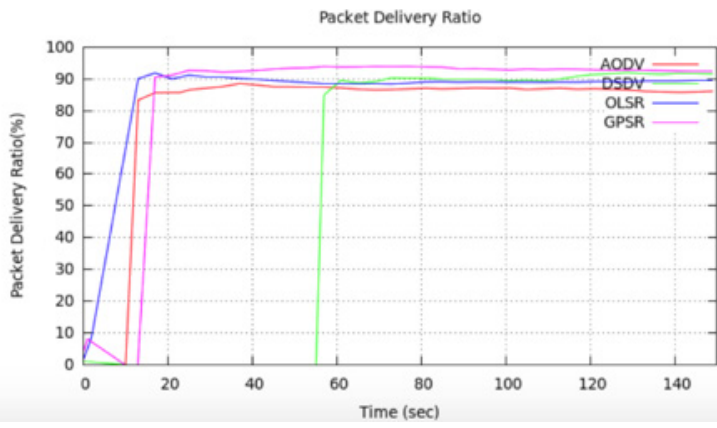

Figure 2. Packet Delivery Ratio

consume time to be updated in the routing tables, which causes the routing loops, ultimately the performance degradation in AODV, OLSR and DSDV. Further, the rapid changes in the topology may cause inappropriate forwarding of packets, due to which the receiver receives duplicate packets in higher quantity. These duplicates increase the success rate of packet delivery ratio in GPSR routing protocol.

\subsection{End to end Delivery}

The end-to-end delay is defined as the average time it takes to a protocol to successfully transmit packets from a source to destination node. Figure 3 presents the average end-to- end delay of AODV, DSDV, OLSR and GPSR protocols with different number of nodes. In all of the plots of Figure 3, AODV and DSDV has longer delay in its performance than OLSR and GPSR routing protocols. AODV is an on-demand protocol, which establishes the connection when required. Due to which, it increases the convergence time. DSDV also consumes time by frequently updating the routing table. For that, the nodes in DSDV wait for such update packets, which causes longer end-to-end delay.

OLSR has the shortest delay compared with all other protocols. The end-to-end delay of OLSR and GPSR decreases with the increase in the number of nodes.

\section{Conclusion}

With the increasing wireless devices the MANETs provide an efficient way for the devices to stay connected to the network. More and more applications use clouds and virtual servers to cope with the routing issues for high performance. The simulation results have clearly shown that the performance of OLSR in terms of throughput is greater than AODV, DSDV and GPSR in the given scenarios. However, the DSDV protocol also improves the throughput when compared with AODV and GPSR. Due to high mobility the performance of GPSR is very low compared to other protocols and specifically in the scenario when the number of nodes increase. AODV slightly improves the throughput, as much as DSDV and OLSR protocols in the scenario where the number of nodes are small. The packet delivery ratio of GPSR remains greater than the other protocols but its overall performance is less than other protocols. The reason behind it is that, the GPSR mostly uses the greedy forwarding strategy for transmission. First, the greedy forwarding strategy is measured as unsuitable for highly mobile networks due the fact that nodes maintain the routing information when they are out of range. This strategy leads to the packet loss. Second, GPSR uses the beacon mechanism, where the information of the location table is affected by the weak signals. In terms of end-to-end delay, 


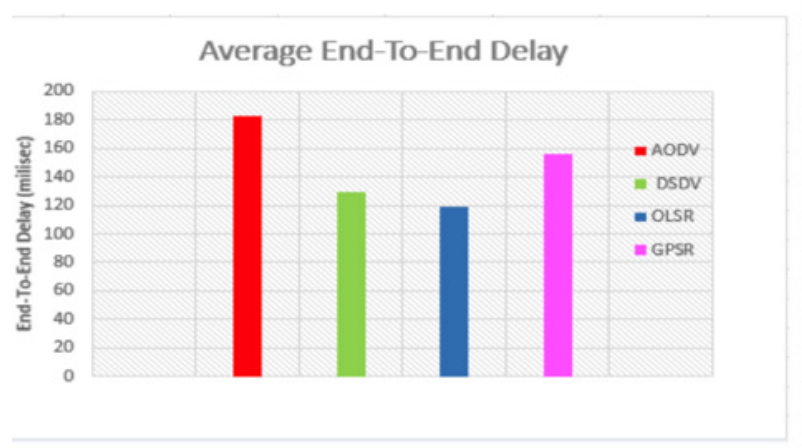

(a) 5 nodes.

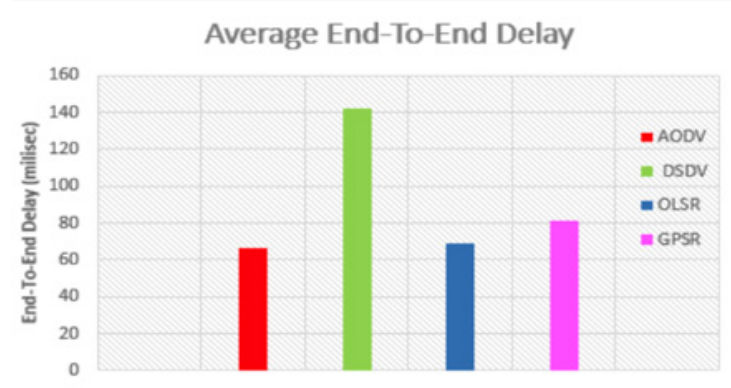

(c) 30 nodes.

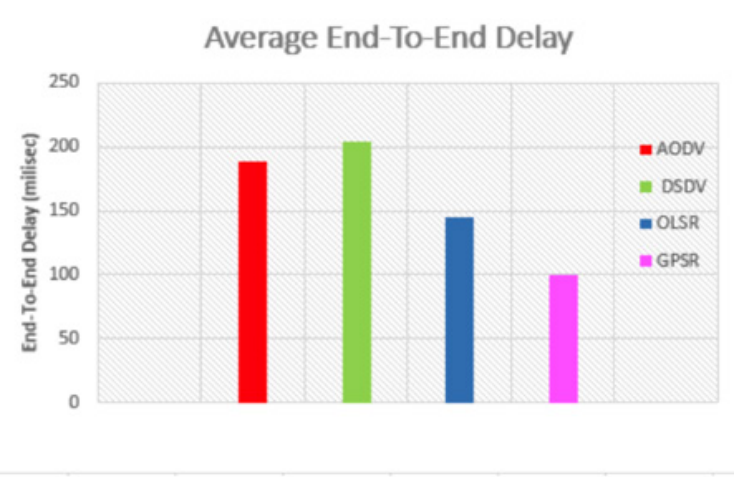

(b) 10 nodes.

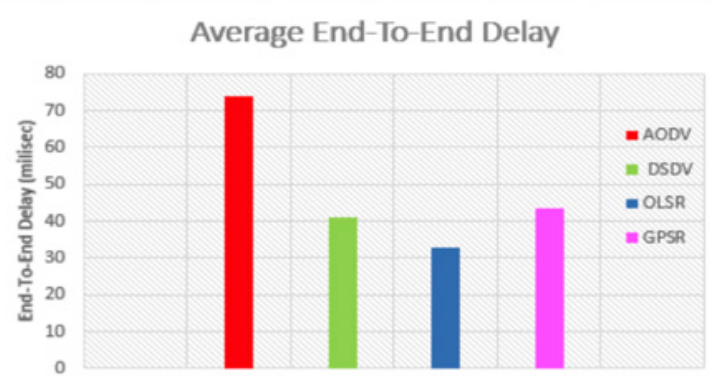

(d) 50 nodes.

Figure 3. Average End-to-Day Delay

AODV and DSDV, the routing is not as efficient as OLSR and GPSR.

On the basis of this study, it is suggested that OLSR is a reliable routing protocol for MANET based applications even with higher number of nodes. The conclusion applies to the mobile nodes in MANETs. In future, due to high mobility in

\section{REFERENCES}

1. AbdulraheemYasir, M. \& Ahmad Zukarnain, Z. (2014). Comparative analysis of AODV, DSDV and GPSR routing protocols in MANET scenarios of real urban area, Australian Journal of Basic and Applied Sciences, 8(15), 145-157.

2. Al-Ani, R. (2011). Simulation and performance analysis evaluation for variant MANET routing protocols, International Journal of Advancements in Computing Technology, 3(1), 1-12.

3. Aujla, G. S. \& Kang, S. S. (2013). Comprehensive Evaluation of AODV, DSR, GRP, OLSR and TORA Routing Protocols with varying number of nodes and traffic applications over MANETs, IOSR Journal of Computer Engineering, 9(3), 54-61.
MANET, a research on the effects of mobility issues is viewed as being important. For that, the analysis of different routing protocols in VANETs is also necessary. Nowadays, the sensing and monitoring applications are boosting and need various technologies such as cloud computing. A further research on the evaluation of routing protocols for the sensors would be a great contribution.

4. Clausen, T. \& Jacquet, P. (2003). Optimized link state routing protocol (OLSR), RFC 3626.

5. Etorban, A., King, P. J. B \& Trinder, P. (2009). In A performance comparison of MDSDV with $A O D V$ and $D S D V$ routing protocols. UK Performance Engineering Workshop.

6. Fotohi, R., Jamali, S. \& Sarkohaki, F. (2013). Performance Evaluation of AODV, LHC-AODV, OLSR, UL-OLSR, DSDV Routing Protocols, International Journal of Information Technology and Computer Science (IJITCS), 5(10), 21.

7. Giordano, S. (2002). Mobile ad hoc networks, Handbook of wireless networks and mobile computing, 325-346. 
8. Goyal, P. (2012). Simulation study of comparative performance of AODV, OLSR, FSR \& LAR, routing protocols in MANET in large scale scenarios. In World Congress on Information and Communication Technologies (WICT). IEEE.

9. Gupta, S. K. \& Saket, R. K. (2011). Performance metric comparison of AODV and DSDV routing protocols in manets using ns-2, International Journal of Research and Reviews in Applied Sciences, 7(3), 339-350.

10. Gupta, V. (2012). Comparative Performance Analysis of AODV, DSR, DSDV, LAR1 and WRP Routing Protocols in MANET using GloMoSim 2. 0. 3 Simulator, International Journal of Computer Applications, 52(20).

11. Jacquet, P. et. al (2001). Optimized link state routing protocol for ad hoc networks. In Multi Topic Conference (INMIC). IEEE.

12. Karp, B. \& Kung, H.-T. (2000). GPSR: Greedy perimeter stateless routing for wireless networks. In Proceedings of the $6^{\text {th }}$ Annual International Conference on Mobile Computing and Networking. ACM.

13. Li, F. \& Wang, Y. (2007). Routing in vehicular adhoc networks: Asurvey, IEEE Vehicular technology magazine, 2(2), 12-22.

14. Morshed, M. M. et al. (2009). Simulation and analysis of ad-hoc on-demand distance vector routing protocol. In Proceedings of the $2^{\text {nd }}$ International Conference on Interaction Sciences: Information Technology, Culture and Human. ACM.

15. Norouzi, A. \& Sertbas, A. (2012). Efficiency Analysis and Comparative Performance Evaluation of Routing Protocols in Mobile Ad Hoc Networks, Studies in Informatics and Control, 21(2), 209-220. ISSN 1220-1766.

16. Pan, Y. (2010). Design routing protocol performance comparison in NS2: AODV comparing to DSR as example. Department of Computer Science SUNY Binghamton, Vestal Parkway East.
17. Patil, V. P. (2012). Reactive and proactive routing protocol performance evaluation for qualitative and quantitative analysis in mobile ad hoc network, International Journal of Scientific and Research Publications, 2(9), 1-8.

18. Perkins, C., Belding-Royer, E. \& and Das, S. (2003). Ad hoc on-demand distance vector (AODV) routing, RFC 3561.

19. Perkins, C. E. \& Bhagwat, P. (1994). Highly dynamic destination-sequenced distancevector routing (DSDV) for mobile computers, ACM SIGCOMM Computer Communication Review, 24(4). ACM.

20. Ramesh, V., Subbaiah, P., Koteswar Rao, N. \& Janardhana Raju, M. (2010). Performance Comparison and Analysis of DSDV and AODV for MANET, International Journal on Computer Science and Engineering, 2(2), 183-188.

21. Ravi, Y. et al. (2012). Position based Routing in Mobile Adhoc Networks, International Journal of Computer Applications, 55(5).

22. Sharma, P., Kalia, A. \& Thakur, J. (2012). Performance analysis of AODV, DSR AND DSDV routing protocols in mobile ad-hoc network (MANET), Journal of Information Systems and Communication, 3(1), 322.

23. Singh, J. \& Mahajan, R. (2013). Performance analysis of AODV and OLSR using OPNET, Int. J. Comput. Trends Technol, 5, 114-117.

24. Tuteja, A., Gujral, R. \& Thalia, S. (2010). Comparative performance analysis of DSDV, AODV and DSR routing protocols in MANET using NS2. In International Conference on Advances in Computer Engineering (ACE), 2010. IEEE.

25. Upadhyay, A. \& Phatak, R. (2013). Performance Evaluation of AODV DSDV and OLSR Routing Protocols with Varying FTP Connections in MANET, IJRCCT, 2(8), 531-535. 